\title{
Factores externos y rasgos estructurales que caracterizan el desarrollo de los cibermedios en América
}

\author{
Elias Said-Hung*, Jorge Valencia-Cobos*
}

\begin{abstract}
Resumen: Este artículo analiza 111 medios digitales de 25 países de todo el continente americano, con el fin de caracterizar y comparar el desarrollo existente en los medios digitales e identificar los aspectos socio-económicos y tecnológicos que distinguen e inciden en estos portales en cada país y región de dicho continente. Este artículo se basa en los resultados hallados en el proyecto «El desarrollo de los medios digitales en el continente americano, España, Portugal y Reino Unido", realizado en 2011 en la Universidad del Norte; además de los indicadores dispuestos por el Banco Mundial sobre cada país, los cuales permiten ver, entre otras cosas, el desarrollo de los medios digitales en toda América, las debilidades que tienen en la profundización, herramientas de interacción y la personalización de la información publicada en estos medios, además de una segmentación del desarrollo de estos medios por regiones, con diferencias sustantivas en cuanto a la concentración demográfica, conectividad y número de abonados a Internet y el porcentaje de población rural, entre otros aspectos, entre los países analizados. Asimismo, se logra establecer como uno de los factores externos que inciden en el tema tratado la densidad de usuarios conectados a Internet.
\end{abstract}

Palabras clave: Medios, digital, periodismo, comunicación, desarrollo, América.

\section{External factors and structural features characterizing the development of digital mass media in the Americas}

Abstract: This article analysed 111 digital mass media sites from 25 countries throughout the Americas, with the purpose of characterising and comparing the development of cyber-media there, and of identifying the socio-economic and technological aspects which distinguish these Web sites in each country and region in the continent. The data analysed in this article were taken from the project "The development of the digital media in the American continent, Spain, Portugal and United Kingdom", undertaken by the Universidad del Norte, and also from different indicators available from the World Bank database for each country. These provide information on the development process of the digital media in the Americas, weaknesses regarding penetration, tools for interaction, and personalization of the information published in these media. The data also reveal the segmentation of this development, according to region, with substantial differences among the countries analysed in such aspects as demographic concentration, Internet

* Universidad del Norte. Barranquilla, Colombia. Correo-e: saide@uninorte.edu.co; jorval2911@ yahoo.es.

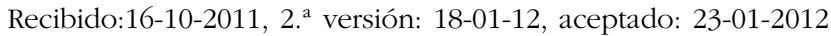


Factores externos y rasgos estructurales que caracterizan el desarrollo de los cibermedios...

connectivity, number of users, and percentage of rural population. One important external factor identified was the density of users connected to the Internet.

Keywords: Mass media, digital, journalism, communication, development, America.

\section{Introducción}

Según Canga (2001), la evolución de Internet hasta ahora se ha caracterizado por la sucesión de tres fases claramente diferenciadas desde su aparición, las cuales el autor denomina: "seducción tecnológica», "seducción económica» y "adecuación de contenidos». Es en esta última fase en donde nos encontramos en la actualidad, pues se revela allí como fundamental el papel que deben tener las empresas de comunicación y los profesionales que allí trabajan en la tarea de adaptación de los nuevos paradigmas empresariales y periodísticos, según las nuevas exigencias del escenario actual digital; este último, caracterizado por una creciente complejidad de la mediamorfosis, o transformación de los medios de comunicación hacia estándares electrónicos (digitales), propuestos por Fidler (1998), hacia un hábitat donde también actúa la infomorfosis, o la transformación de la información y los códigos de comunicación (Flores, 2011).

El actual escenario nos permite trazar algunos de los nuevos rasgos dentro unas sociedades contemporáneas en las que la integración de lecturas lineales y no lineales, la producción de contenidos cada vez más multimedia y la creciente fragmentación de una audiencia, cada vez más desterritorializada, autónoma y afín al "prosumidor» de Toffler (1980), marcan los retos que hoy hacen que muchos investigadores orienten sus análisis de la sociedad de la información, las comunicaciones, los medios, los periodistas digitales y el estudio de los factores que pueden estar incidiendo en estos procesos o contextos profesionales.

Uno de los rasgos que le caracteriza a la nueva etapa multimedia es la capacidad de aproximación de espacios naturales alrededor de los medios de comunicación; ya que están dotados de un alto nivel de conectividad entre sí (Cabrera, 2001). Por lo expuesto hasta el momento, el sector de los medios digitales constituye uno de los principales puntos de referencia para la gestión de los flujos caóticos de la información y contribuye en la formación de nuevas agendas, matrices de opinión pública y vínculos complejos en la estructura usuario-medio-usuarios.

\section{Hacia una medición unificada del desarrollo de los medios digitales}

Según Rodríguez-Martínez y otros (2010), los medios digitales pueden ser medidos de acuerdo a un conjunto de indicadores: 1) generales, que miden la accesibilidad del sitio Web, su visibilidad y popularidad, entre otros aspectos relacionados con los aspectos que toda Web de un diario digital debe cuidar; 2) específicos internos, que dan cuenta de la profundización de la información, las 
herramientas de interacción empleadas en los medios digitales, así como la personalización de la información y de aquellos elementos que el sitio de un diario digital debiese incluir, y 3) específicos externos, relacionados con el impacto de las Web sociales en los medios digitales, en el que se miden los mecanismos de fidelización de los usuarios, a través de las Web 2.0 y otras herramientas dispuestas en los medios digitales, así como los mecanismo de difusión de los contenidos generados en el diario digital. Esta propuesta de medición, si bien resulta un avance a favor de la comprensión más clara del actual estado del desarrollo de los medios digitales, así como la verificación empírica de las propuestas teóricometodológicas que se vienen adelantando en la literatura científica (Díaz-Noci, 1997; Van Der Wurff y Lauf, 2005; Zamith, 2008; Palacios y Díaz-Noci, 2009; Flores, 2008, 2011; o García de Torres y otros, 2011, entre otros), carece de un planteamiento que permita sintetizar en un único indicador o medida el estado de avance que pudiesen presentar estos medios. Es así como, tomando como base la propuesta metodológica de medición de los medios digitales de estos autores, Said-Hung y otros (2011) han propuesto un índice que pueda ayudar a: 1) dueños y periodistas de los medios digitales a establecer una clara noción en torno a dónde se encuentran y los aspectos que ameritan ser revisados o fortalecidos por estos para el abordaje más firme de los retos económicos y profesionales dentro de este sector en la actualidad; y en segundo lugar poder elaborar estudios que permitan establecer los diferentes factores, internos y externos a este sector, que pueda estar incidiendo en el desarrollo del mismo.

Si bien ha sido una marca distintiva en los estudios adelantados por autores ya citados aquí, y otros como McCombs y otros (1991), Turner (2005), Lewis y Fabos (2005), Costanza-Chock (2008), Hart (2009), Resina (2010), o Boyer (2010), por citar algunos, el abordaje de interesantes y valiosas reflexiones en torno al impacto de los medios de comunicación en la formación de la opinión pública, aun resulta prioritario la falta de bases investigativas y conceptuales que hoy pudiesen ayudar a responder a inquietudes como: ¿Existen diferencias significativas entre el desarrollo de los medios digitales y el desarrollo de los países? ¿Qué características diferencian el desarrollo de los medios digitales en este continente? ¿Qué factores externos a los medios digitales tienen mayor peso sobre la evolución de este sector?

Si bien los medios digitales y los profesionales que en ellos trabajan, viven y son dinamizadores (en primera persona) de las transformaciones generadas por el auge de la cibercultura (Piscitelli, 2002), y la Sociedad de la Información (Cardoso, 2008 y Castells, 2000), entre otros procesos relacionados, los aspectos externos (sociales) deberían ser considerados también para el requerido análisis sobre este fenómeno.

\section{Metodología}

La metodología empleada para el tratamiento del tema propuesto tomó en consideración lo planteado por Rodríguez-Martínez y otros (2010) y Said-Hung y 
otros (2011), al momento de abordar dos objetivos generales: analizar y medir el nivel de desarrollo de los medios digitales y de las Web 2.0 en los 25 países del continente americano; así como, estimar cuáles son los factores tecnológicos, socio-demográficos, económicos y profesionales que pueden estar incidiendo en el desarrollo de los medios digitales en los países de dicha región. Para lograr ambos objetivos, el conjunto de los datos mostrados y analizados en esta investigación será articulado en torno a un índice de desarrollo, el cual servirá al momento de comprender y ubicar a América en torno al proceso de digitalización de los medios de comunicación. Este estudio se realizó sobre los medios digitales de mayor acceso y visibilidad dentro del ranking Top100 de Alexa (http:// www.Alexa.com ) en cada uno de los países de seleccionados para este proyecto (véase tabla I).

Ante el carácter abierto que tiene el debate y la diversidad de propuestas de medición de los medios digitales en la actualidad, como por ejemplo los expuestos por Palacios (2011), la metodología aplicada para el abordaje exploratorio del tema propuesto aquí se hizo sobre la base de la complejidad que reviste la resolución del tema e interrogantes planteados en este artículo. Todo ello, además del carácter transversal que brinda ésta investigación al momento de hacer una aproximación a algunos de los principales rasgos que marcan, desde nuestro punto de vista, el desarrollo existente en la actualidad en los medios digitales, en torno a tres dimensiones: 1) el carácter estructural observado en los portales, que marcan el diseño y acceso de estos a los usuarios; 2) los rasgos microestructurales observados dentro de los contenidos y el propio medio, al momento de hacer un uso de los recursos TIC para el aumento de la profundización de la información, el uso de herramientas interactivas para el aumento de las dinámicas de comunicación y divulgación entre el medio-periodista-usuario y en la personalización de los contenidos, de acuerdo a los intereses y necesidades informativas de cada usuario, y 3) los niveles de apropiación, tanto en la divulgación y difusión de los contenidos, por parte de los usuarios y el propio medio analizado, a través de las Web 2.0.

El trabajo de investigación que presentamos ofrece dos unidades de observación diferenciadas: el medio (en el que se estudiarán tanto los aspectos generales y específicos, tanto del portal Web de los medios analizados, como de los contenidos publicados en la página principal de éstos al momento de realizarse el levantamiento de los datos pautados en este trabajo) y el entorno (en el que se identificarán los rasgos socio-demográficos, económicos y tecnológicos existentes en los países analizados al momento de realizar este proyecto).

Los resultados obtenidos del proyecto del que parte este artículo se articularán en torno a un índice de desarrollo de medios digitales, sintetizado a partir de la siguiente fórmula: DC-Ame (Desarrollo de medios digitales en países del continente americano $)=I G$ (Indicadores generales $)+I E i$ (Indicadores específicos internos) + IExt (Indicadores externos - Web 2.0).

La totalidad de los apartados que forman parte del DC-Ame dará un valor $X$ sobre 100 puntos, correspondientes al total de puntos pautados para la medición. 


\section{TABLA I}

Cibermedios analizados, según nivel de visibilidad de Alexa. Febrero de 2011

\begin{tabular}{|c|c|c|}
\hline País & Medio analizado & URL \\
\hline Argentina & $\begin{array}{l}\text { Perfil, Clarín, Minuto Uno, } \\
\text { Página 12, La Nación }\end{array}$ & $\begin{array}{l}\text { http://www.perfil.com/, http://www.minutouno. } \\
\text { com.ar/, http://www.pagina12.com.ar/, http:// } \\
\text { www.lanacion.com.ar/ }\end{array}$ \\
\hline Bahamas & $\begin{array}{l}\text { The Bahamas Weekly, Baha- } \\
\text { mas Press, The Nassau Guar- } \\
\text { dian, Tribune } 242\end{array}$ & $\begin{array}{l}\text { http://www.thebahamasweekly.com/, http://ba- } \\
\text { hamaspress.com/, http://www.thenassauguar- } \\
\text { dian.com/, http://www.tribune242.com/ }\end{array}$ \\
\hline Bolivia & $\begin{array}{l}\text { Los Tiempos, La Razón, El } \\
\text { Deber, El Diario, El Día }\end{array}$ & $\begin{array}{l}\text { http://www.lostiempos.com/, http://www.la-ra- } \\
\text { zon.com/, http://www.eldeber.com.bo/, http:// } \\
\text { www.eldiario.net/, http://eldia.com.bo/ }\end{array}$ \\
\hline Brasil & O Globo, Clicrbs & $\begin{array}{l}\text { http://oglobo.globo.com/, http://www.clicrbs. } \\
\text { com.br/ }\end{array}$ \\
\hline Canadá & $\begin{array}{l}\text { TSN, CBC, The Globe and } \\
\text { Mail, Cnews Canoe, Cyber } \\
\text { Presse }\end{array}$ & $\begin{array}{l}\text { http://www.tsn.ca/, http://www.cbc.ca/, http:// } \\
\text { www.theglobeandmail.com/, http://cnews.canoe. } \\
\text { ca/, http://www.cyberpresse.ca/ }\end{array}$ \\
\hline Chile & $\begin{array}{l}\text { La Tercera, La Cuarta, Coo- } \\
\text { perativa, Las Últimas Noti- } \\
\text { cias, El Mercurio }\end{array}$ & $\begin{array}{l}\text { http://latercera.com/, http://www.lacuarta.cl/, } \\
\text { http://www.cooperativa.cl/, http://www.lun.com/, } \\
\text { http://www.emol.com/ }\end{array}$ \\
\hline Colombia & $\begin{array}{l}\text { El Tiempo, Semana, El Es- } \\
\text { pectador, Portafolio, El Co- } \\
\text { lombiano }\end{array}$ & $\begin{array}{l}\text { http://www.eltiempo.com/, http://www.semana. } \\
\text { com/, http://www.elespectador.com/, http:// } \\
\text { www.portafolio.co/, http://www.elcolombiano. } \\
\text { com/ }\end{array}$ \\
\hline Costa Rica & $\begin{array}{l}\text { Nación, Al Día, Teletica, Dia- } \\
\text { rio Extra, Evardo Herrera }\end{array}$ & $\begin{array}{l}\text { http://www.nacion.com/, http://www.aldia.cr/, } \\
\text { http://www.teletica.com/, http://www.diarioextra. } \\
\text { com/, http://everardoherrera.com/ }\end{array}$ \\
\hline Cuba & $\begin{array}{l}\text { Cuba Debate, Juventud Re- } \\
\text { belde, Escambray, Gramma, } \\
\text { Prensa Latina }\end{array}$ & $\begin{array}{l}\text { http://www.cubadebate.cu/, http://www.juventu- } \\
\text { drebelde.cu/, http://www.escambray.cu/, http:// } \\
\text { www.granma.cubaweb.cu/, http://www.prensa- } \\
\text { latina.cu/ }\end{array}$ \\
\hline Ecuador & $\begin{array}{l}\text { Comercio, Hoy, Ecuador In- } \\
\text { mediato, El universo, Ecua- } \\
\text { dor en vivo }\end{array}$ & $\begin{array}{l}\text { http://www.elcomercio.com/, http://hoy.com.ec/, } \\
\text { http://www.ecuadorinmediato.com/, http://www. } \\
\text { eluniverso.com/, http://www.ecuadorenvivo. } \\
\text { com/ }\end{array}$ \\
\hline El Salvador & $\begin{array}{l}\text { El Salvador, La Prensa Gráfi- } \\
\text { ca, La Página, Diario Latino }\end{array}$ & $\begin{array}{l}\text { http://www.elsalvador.com/, http://www.lapren- } \\
\text { sagrafica.com/, http://lapagina.com.sv/, http:// } \\
\text { diariocolatino.com/ }\end{array}$ \\
\hline $\begin{array}{l}\text { Estados } \\
\text { Unidos }\end{array}$ & $\begin{array}{l}\text { NY Times, CNN, ESPN, Huff- } \\
\text { ington Post, Fox News }\end{array}$ & $\begin{array}{l}\text { http://www.nytimes.com/, http://edition.cnn. } \\
\text { com/, http://espn.go.com/, http://www.huffing- } \\
\text { tonpost.com/, http://www.foxnews.com/ }\end{array}$ \\
\hline Guatemala & $\begin{array}{l}\text { Siglo 21, El Periódico, Nues- } \\
\text { tro Diario, Prensa Libre }\end{array}$ & $\begin{array}{l}\text { http://www.s21.com.gt/, http://elperiodico.com. } \\
\text { gt/, http://www.nuestrodiario.com/, http://pren- } \\
\text { salibre.com/ }\end{array}$ \\
\hline
\end{tabular}


TABLA I (continuación)

\begin{tabular}{|c|c|c|}
\hline País & Medio analizado & URL \\
\hline Honduras & $\begin{array}{l}\text { La Prensa, Diario Deportivo } \\
\text { Diez, El Heraldo, Tiempo, La } \\
\text { Tribuna }\end{array}$ & $\begin{array}{l}\text { http://www.laprensa.hn/, http://www.diez.hn/, } \\
\text { http://elheraldo.hn/, http://www.tiempo.hn/, } \\
\text { http://www.latribuna.hn/ }\end{array}$ \\
\hline Jamaica & $\begin{array}{l}\text { Jamaica Gleaner, Jamaica } \\
\text { Observer, JIS }\end{array}$ & $\begin{array}{l}\text { http://jamaica-gleaner.com/, http://www.jamaica- } \\
\text { observer.com/, http://www.jis.gov.jm/ }\end{array}$ \\
\hline México & $\begin{array}{l}\text { El Universal, Medio Tiempo, } \\
\text { Milenio, Reforma, El Norte }\end{array}$ & $\begin{array}{l}\text { http://www.eluniversal.com.mx/, http://msn.me- } \\
\text { diotiempo.com/, http://www.milenio.com/, http:// } \\
\text { www.reforma.com/, http://www.elnorte.com/ }\end{array}$ \\
\hline Nicaragua & $\begin{array}{l}\text { El Nuevo Diario, La Prensa, } \\
\text { Fútbol Nica }\end{array}$ & $\begin{array}{l}\text { http://www.elnuevodiario.com.ni/, http://www. } \\
\text { laprensa.com.ni/, http://www.futbolnica.net/ }\end{array}$ \\
\hline Panamá & $\begin{array}{l}\text { Prensa, Telemetro, TVN, } \\
\text { Qué Pasa Panamá, Panamá } \\
\text { América }\end{array}$ & $\begin{array}{l}\text { http://www.prensa.com/, http://www.telemetro. } \\
\text { com/, http://www.tvn-2.com/, http://quepasapa- } \\
\text { nama.com/, http://www.panamaamerica.com.pa/ }\end{array}$ \\
\hline Paraguay & $\begin{array}{l}\text { La Nación, ABC, Última } \\
\text { Hora, Paraguay, Crónica }\end{array}$ & $\begin{array}{l}\text { http://www.lanacion.com.py/, http://www.abc. } \\
\text { com.py/, http://www.ultimahora.com/, http:// } \\
\text { www.paraguay.com/, http://www.cronica.com.py/ }\end{array}$ \\
\hline Perú & $\begin{array}{l}\text { Perú, El Comercio, La Repú- } \\
\text { blica, RPP, Perú } 21\end{array}$ & $\begin{array}{l}\text { http://peru.com/, http://elcomercio.pe/, http:// } \\
\text { www.larepublica.pe/, http://www.rpp.com.pe/, } \\
\text { http://peru21.pe/ }\end{array}$ \\
\hline Puerto Rico & $\begin{array}{l}\text { El Nuevo Día, Vocero, Pri- } \\
\text { mera Hora }\end{array}$ & $\begin{array}{l}\text { http://www.elnuevodia.com/, http://www.vocero. } \\
\text { com/, http://www.primerahora.com/ }\end{array}$ \\
\hline $\begin{array}{l}\text { República } \\
\text { Dominicana }\end{array}$ & $\begin{array}{l}\text { Diario Libre, El Nacional, El } \\
\text { Nuevo Diario, Listín Diario }\end{array}$ & $\begin{array}{l}\text { http://www.diariolibre.com/, http://elnacional. } \\
\text { com.do/, http://elnuevodiario.com.do/, http://lis- } \\
\text { tindiario.com/ }\end{array}$ \\
\hline $\begin{array}{l}\text { Trinidad y } \\
\text { Tobago }\end{array}$ & $\begin{array}{l}\text { Guardian, Trinidad Express, } \\
\text { Newsday }\end{array}$ & $\begin{array}{l}\text { http://www.guardian.co.tt/, http://www.trinida- } \\
\text { dexpress.com/, http://newsday.co.tt/ }\end{array}$ \\
\hline Uruguay & $\begin{array}{l}\text { El País, Observador, Ovación } \\
\text { Digital, Montevideo, La Re- } \\
\text { pública }\end{array}$ & $\begin{array}{l}\text { http://www.elpais.com.uy/, http://www.elobserva- } \\
\text { dor.com.uy/, http://www.ovaciondigital.com.uy/, } \\
\text { http://www.montevideo.com.uy/, http://www.lr21. } \\
\text { com.uy/ }\end{array}$ \\
\hline Venezuela & $\begin{array}{l}\text { El Nacional, El Universal, } \\
\text { Noticiero Digital, Noticias } 24 \text {, } \\
\text { Meridiano }\end{array}$ & $\begin{array}{l}\text { http://www.el-nacional.com/, http://www.eluni- } \\
\text { versal.com/, http://www.noticierodigital.com/, } \\
\text { http://www.noticias24.com/, http://www.meridia- } \\
\text { no.com.ve/ }\end{array}$ \\
\hline
\end{tabular}

Fuente: elaborada por los autores.

El peso de cada apartado variará en relación con el número de variables pautadas en cada uno, distribuyéndose los 100 puntos según el peso previamente determinado para los tres grupos de indicadores considerados para la medición de los niveles de desarrollo de los cibermedios. 
Los DC-Ame se clasificaron en 3 niveles de desarrollo (Nivel 1 (DC-Ame < 40), Nivel 2 (DC-Ame > $40<60$ ), Nivel 3 (DC-Ame > 60), a partir de la distribución de frecuencias de los puntajes obtenidos por cada medio analizado en este artículo, y se agruparon los países según su ubicación geográfica (Norteamérica, Centroamérica, Suramérica, Caribe (islas). Una vez realizada esta transformación se utilizó la técnica de análisis factorial de correspondencias simples, con el objetivo de identificar las posibles relaciones existentes entre las características regionales y los niveles de IDC, sobre un mapa factorial, donde los puntos más cercanos reflejan las asociaciones entre categorías.

Al momento de intentar establecer los aspectos externos que caracterizan a los países analizados en relación con el nivel de desarrollo de los medios digitales analizados en ellos, se empleó la base de datos de indicadores del Banco Mundial dispuesta por cada uno de los 25 países analizados (http://datos.bancomundial.org/pais), tomando como referente el 2008 y de acuerdo con los siguientes criterios: el carácter no inmediato que pueden ejercer los aspectos sociales (externos) en el interior del proceso de evolución de los medios digitales en cada país, la distribución temática establecida para la consideración de lo aquí tratado (demografía, economía, educación, empleo, infraestructura, tecnología y salud), así como la disponibilidad unificada (en los 25 países analizados) de los mismos indicadores para su consideración.

A partir de los datos obtenidos del DC-Ame propuesto y del conjunto de indicadores del Banco Mundial tomados en consideración, se evaluó la posible relación entre estos, así como su magnitud. Para lo anterior se procedió a la definición del modelo de regresión, de la forma $Y=B_{1} X_{1}+B_{2} X 2 \ldots, B_{n} X n$; introduciendo las variables paso por paso valiéndose del programa Pasw 18.

\section{Resultados}

Los resultados obtenidos durante el mes de abril y mayo de 2011 nos permiten disponer de un ranking de calidad de los medios digitales de todo el continente americano que vislumbre su grado de desarrollo presentado a nivel continental. El peso de cada apartado que da cuenta del DC-Ame varió, con relación al número de variables pautadas en cada uno y se distribuyeron los 100 puntos como se muestra a continuación:

- Indicadores generales (IG): 27 puntos máximos, los cuales valorarán aspectos como los errores de accesibilidad de los diarios digitales analizados, la visibilidad y popularidad de los diarios digitales y la presencia o no de buscadores simples, de buscadores avanzados, hemeroteca y mapa del sitio Web en cada uno de los medios digitales analizados en este trabajo.

- Indicadores específicos internos (IEi): 50 puntos, que medirán aspectos como la profundización de la información, el nivel de uso de herramientas de interacción en los diarios digitales analizados y la capacidad de perso- 
nalización de la información expuesta en los diarios digitales analizados; como por ejemplo, la presencia de alertas, RSS, y envío de noticias por email, entre otros.

- Indicadores externos (IExt): 23 puntos, donde se medirán aspectos relacionados con el uso de las Web 2.0.

La tabla II nos permite ver, en primer lugar, los medios digitales de principal desarrollo en toda América, según el DC-Ame propuesto, liderados por: El Tiempo de Colombia (77/100 puntos), The NY Times de Estados Unidos (77/100 puntos), El Mercurio de Chile (75/100 puntos) y El País del Uruguay (75/100 puntos). Estos medios digitales líderes se encuentran contrapuestos con lo observado en el Diario Extra de Costa Rica (22/100 puntos), El Diario de Bolivia (26/100), Trinidad Newsday (27/100 puntos), las Últimas Noticias de Chile (32/100 puntos) y Fútbol Nica de Nicaragua (36/100 puntos), ubicados respectivamente en los últimos puestos del nivel de desarrollo de la temática aquí tratada.

Si tomamos como referencia los valores encontrados en la tabla II, vemos cómo los medios digitales analizados se caracterizan, en 2011, por una debilidad marcada en aquellos aspectos relacionados con las capacidades de aprovechamiento de las TIC, a favor de la profundización de la información, las herramientas de interacción y personalización de los contenidos desarrollados y difundidos desde estos medios de comunicación digitales. Asimismo, se aprecia como también se requieren avances, aunque en menor proporción, de la accesibilidad, la visibilidad y el acceso a la información de los medios digitales, los cuales aun parecen requerir una revisión y ajuste técnico y de diseño aplicado en dichos portales, que facilite un mayor acceso, directo o a través de los buscadores Web (Google y Yaboo! Search, entre otros), de los contenidos desarrollados y divulgados desde estos medios.

Uno de los aspectos que más fortalezas presentan los medios digitales analizados es el uso de las Web 2.0, tanto facilitando a los usuarios mecanismos para compartir o «llevarse» los contenidos expuestos en estos medios, como generando mecanismos alternativos de exposición de estos, por ejemplo, desde canales Web 2.0 abiertos que suelen ser aplicados, de forma generalizada, desde éstos medios digitales.

Los diferentes rasgos expuestos hasta ahora irán presentándose de forma más negativa a medida que se avance en cada uno de los restantes 91 medios analizados en el proyecto del que parte este artículo (81,98\% de la totalidad de casos estudiados en este artículo), al presentar 10 a 55 puntos de diferencia negativa con los medios digitales estudiados, que lideran este proceso a nivel continental. Es así que se mantienen como aspectos más críticos los señalados previamente de acuerdo con los datos mostrados en la tabla II.

Si tomamos en consideración los niveles de desarrollo de los medios digitales en el continente americano analizados (111 medios), según la media ( $\mu$ ) obtenida a nivel de cada país, región, y todo el continente (tabla III), vemos cómo los países en Norte y Suramérica, respectivamente, son los que se encuentran en 


\begin{tabular}{|c|c|c|c|c|c|c|c|c|c|c|c|c|c|c|c|}
\hline & & 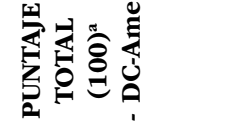 & $\mathbf{R}$ & $\mathfrak{R}$ & $n$ & $n$ & $\stackrel{n}{r}$ & $\stackrel{n}{r}$ & $\stackrel{n}{r}$ & $\stackrel{N}{N}$ & $\mathbb{N}$ & $N$ & $\mathfrak{N}$ & $\mathfrak{N}$ & $r$ \\
\hline & 掿 & 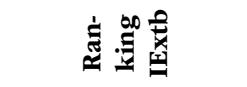 & $\neg$ & - & - & $\neg$ & - & $\neg$ & $\neg$ & $\neg$ & $\neg$ & $\neg$ & $\neg$ & $\neg$ & $\neg$ \\
\hline $\bar{J}$ & 吾 & ซै & $\approx$ & $\approx$ & $\approx$ & $\approx$ & $\approx$ & $\approx$ & 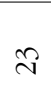 & $\approx$ & $\approx$ & $\approx$ & $\widetilde{\sim}$ & $\approx$ & $\approx$ \\
\hline$\frac{3}{3}$ & 空 & 昆寻 & - & $\sim$ & $N$ & $n$ & $\checkmark$ & in & in & $N$ & 6 & $\wedge$ & $N$ & 0 & $\psi$ \\
\hline$\frac{8}{8}$ & 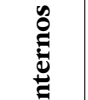 & 这舒 & $\approx$ & $\vec{n}$ & $\vec{n}$ & గి & Әे & $\stackrel{\sim}{\sim}$ & $\stackrel{\infty}{\sim}$ & $\vec{n}$ & $\hat{\imath}$ & $\stackrel{v}{ }$ & $\vec{n}$ & $\hat{\imath}$ & ৯े \\
\hline$\frac{\sqrt{2}}{5}$ & $\overbrace{\mathscr{U}}^{\infty}$ & 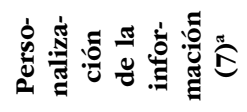 & $r$ & in & 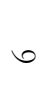 & 0 & 6 & 0 & $r$ & 0 & $n$ & in & 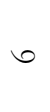 & in & 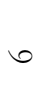 \\
\hline$\stackrel{\infty}{\infty}$ & 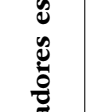 & 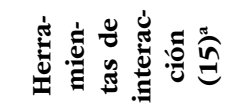 & $\exists$ & 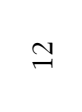 & $\bullet$ & $\stackrel{ }{-}$ & $a$ & $r$ & $a$ & 0 & $a$ & 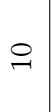 & $\infty$ & $\infty$ & $\stackrel{ }{\sim}$ \\
\hline$\frac{5}{3}$ & 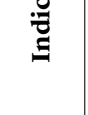 & 起密 & $\begin{array}{l}\text { in } \\
n \\
n\end{array}$ & $\begin{array}{l}\stackrel{8}{n} \\
\stackrel{n}{n}\end{array}$ & $\begin{array}{l}0 \\
12 \\
\infty \\
-1\end{array}$ & $\begin{array}{l}n \\
n \\
n\end{array}$ & $\begin{array}{l}8 \\
\pm \\
\pm\end{array}$ & $\begin{array}{l}8 \\
10 \\
7\end{array}$ & $\begin{array}{l}8 \\
\text { i } \\
7\end{array}$ & $\begin{array}{l}0 \\
n \\
\infty \\
\infty\end{array}$ & $\begin{array}{l}8 \\
\text { in }\end{array}$ & $\begin{array}{l}8 \\
\exists\end{array}$ & $\begin{array}{l}8 \\
8 \\
-1\end{array}$ & $\begin{array}{l}\text { ñ } \\
\text { nn }\end{array}$ & $\begin{array}{l}0 \\
\text { in } \\
\text { I }\end{array}$ \\
\hline ఏे & & 昰 & $n$ & N & 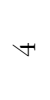 & $n$ & 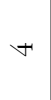 & $n$ & $n$ & 0 & $n$ & $v$ & $r$ & $n$ & in \\
\hline $\begin{array}{l}\tilde{8} \\
\text { s }\end{array}$ & 象 & 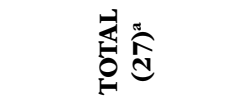 & $\tilde{\text { N }}$ & $\approx$ & $\vec{\sim}$ & $\mathfrak{N}$ & $\vec{\sim}$ & ปี & הี & $\curvearrowright$ & $\tilde{N}$ & $\approx$ & $\stackrel{\infty}{\sim}$ & $\widetilde{N}$ & ¿ \\
\hline$\frac{\pi}{\sqrt[0]{0}}$ & 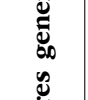 & 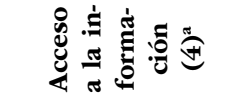 & $\forall$ & $N$ & $n$ & $v$ & $n$ & $\neg$ & $n$ & $v$ & $\sim$ & 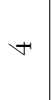 & $n$ & $\sim$ & $\forall$ \\
\hline$\frac{\pi}{0}$ & $\frac{\bar{z}}{\tilde{z}}$ & 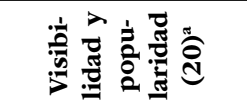 & 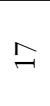 & ㄱ. & 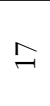 & $\curvearrowright$ & $\Xi$ & $\stackrel{\infty}{\sim}$ & $\stackrel{\infty}{\sim}$ & $\stackrel{?}{\sim}$ & $\Xi$ & $\stackrel{\infty}{\sim}$ & $\underset{\sim}{ \pm}$ & $\curvearrowright$ & $\stackrel{12}{\sim}$ \\
\hline \begin{tabular}{l}
\multirow{\imath}{*}{} \\
\multirow{2}{*}{}
\end{tabular} & & 客焉 & - & - & - & - & - & $n$ & - & - & $n$ & - & - & - & $\neg$ \\
\hline$\stackrel{2}{2}$ & & 党 & $\frac{\frac{\pi}{0}}{\frac{.}{0}}$ & 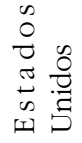 & $\frac{\mathscr{U}}{\bar{\Xi}}$ & 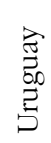 & $\begin{array}{l}\frac{\pi}{0} \\
\frac{0}{0} \\
0 \\
0\end{array}$ & $\begin{array}{l}\vec{D} \\
2 \\
2\end{array}$ & $\begin{array}{l}\frac{\pi}{0} \\
\frac{0}{0} \\
\frac{0}{0}\end{array}$ & Du & $\begin{array}{l}\vec{D} \\
\overrightarrow{2}\end{array}$ & 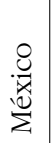 & $\begin{array}{l}\frac{\pi}{0} \\
\frac{.}{0} \\
\frac{0}{0} \\
ن\end{array}$ & 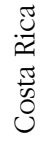 & 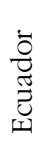 \\
\hline & & 豖 & 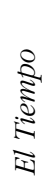 & 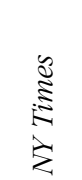 & 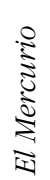 & 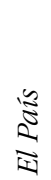 & 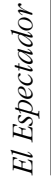 & $\frac{\sqrt{0}}{\sqrt[0]{0}}$ & 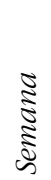 & 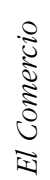 & 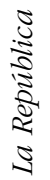 & 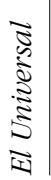 & $\frac{\sqrt{0}}{\frac{2}{2}}$ & $\begin{array}{c}5 \\
0 \\
0 \\
0 \\
0 \\
0 \\
0 \\
0 \\
0\end{array}$ & 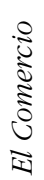 \\
\hline
\end{tabular}


Factores externos y rasgos estructurales que caracterizan el desarrollo de los cibermedios...

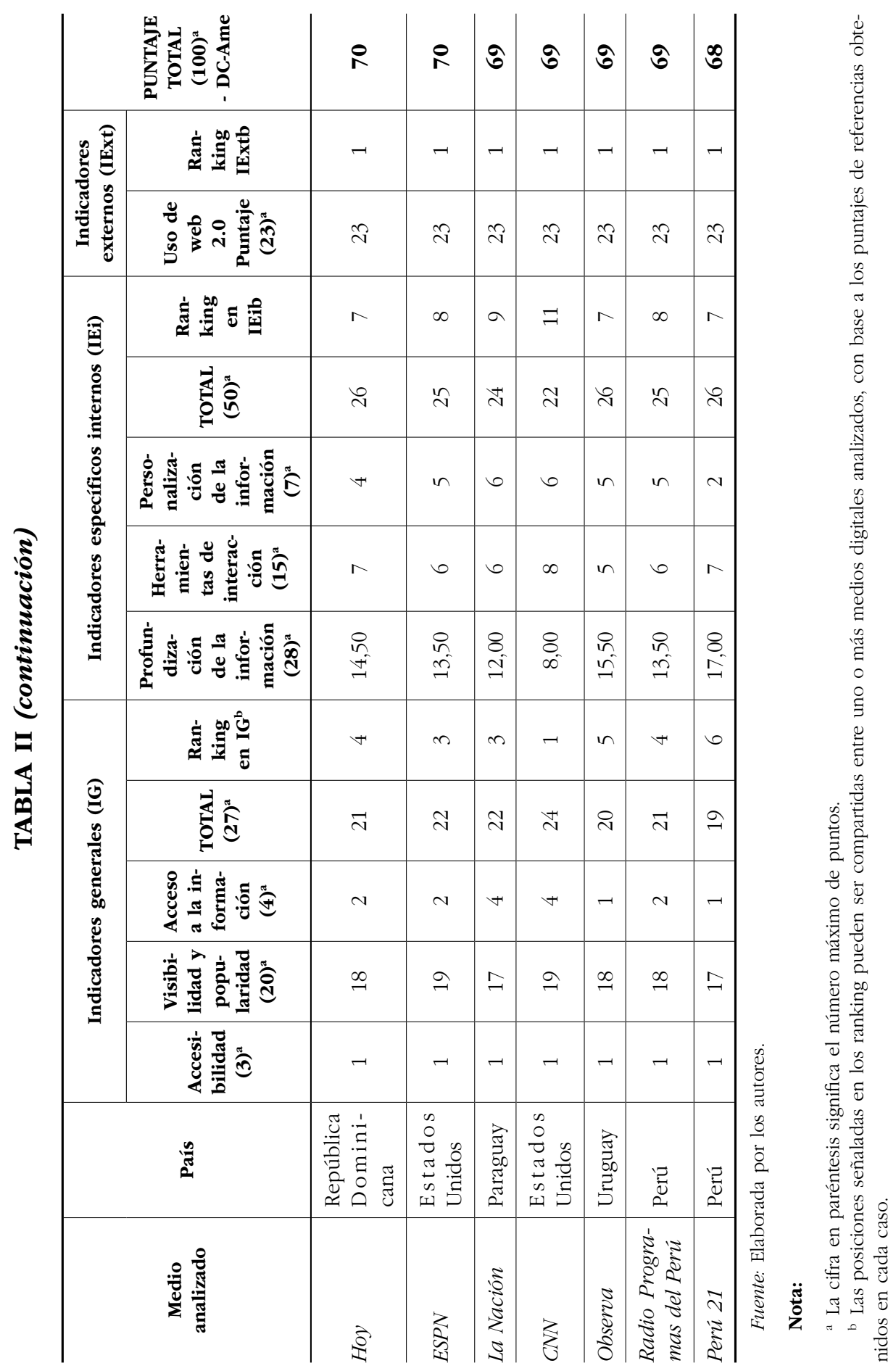




\section{TABLA III}

Nivel de desarrollo y media del DC-AME por país, región y continente americano

\begin{tabular}{|c|c|c|c|c|}
\hline País & Región & $\begin{array}{c}\mu \text { y nivel^} \\
\text { total DC- } \\
\text { Ame } \text { A }^{\wedge} \text { por } \\
\text { país analizado* }\end{array}$ & $\begin{array}{c}\boldsymbol{\mu} \text { y } \text { nivel^ }^{\wedge} \text { total } \\
\text { DC-Ame por } \\
\text { región } \\
\text { analizada*** }\end{array}$ & $\begin{array}{c}\boldsymbol{\mu} \text { y nivel^ total } \\
\text { DC-Ame en el } \\
\text { continente } \\
\text { americano*** }\end{array}$ \\
\hline Estados Unidos & \multirow[b]{3}{*}{ Norteamérica } & 69,4 (Nivel 3) & \multirow[t]{3}{*}{ 65,5 (Nivel 3) } & \multirow[t]{15}{*}{ 59,5 (Nivel 2) } \\
\hline Canadá & & 61,7 (Nivel 3) & & \\
\hline México & & 65,3 (Nivel 3) & & \\
\hline Bahamas & \multirow[b]{6}{*}{ Caribe (islas) } & 44,1 (Nivel 2) & \multirow[t]{6}{*}{54,8 (Nivel 2) } & \\
\hline Cuba & & 58,7 (Nivel 2) & & \\
\hline Jamaica & & 57 (Nivel 2) & & \\
\hline República Dominicana & & 60,9 (Nivel 3) & & \\
\hline Puerto Rico & & 59,8 (Nivel 2) & & \\
\hline Trinidad y Tobago & & 48,5 (Nivel 2) & & \\
\hline Costa Rica & \multirow[b]{6}{*}{ Centroamérica } & 50,1 (Nivel 2) & \multirow[t]{6}{*}{56,2 (Nivel 2) } & \\
\hline El Salvador & & 54 (Nivel 2) & & \\
\hline Guatemala & & 57,3 (Nivel 2) & & \\
\hline Honduras & & 62,3 (Nivel 3) & & \\
\hline Nicaragua & & 55,5 (Nivel 2) & & \\
\hline Panamá & & 57,7 (Nivel 2) & & \\
\hline Argentina & \multirow[b]{10}{*}{ Suramérica } & 60 (Nivel 2) & \multirow[t]{10}{*}{ 61,5 (Nivel 3) } & 59,5 (Nivel 2) \\
\hline Bolivia & & 50,5 (Nivel 2) & & \\
\hline Brasil & & 62,5 (Nivel 3) & & \\
\hline Chile & & 58 (Nivel 2) & & \\
\hline Colombia & & 72,3 (Nivel 3) & & \\
\hline Ecuador & & 59,1 (Nivel 2) & & \\
\hline Paraguay & & 61,1 (Nivel 3) & & \\
\hline Perú & & 70,7 (Nivel 3) & & \\
\hline Uruguay & & 64,7 (Nivel 3) & & \\
\hline Venezuela & & 56 (Nivel 2) & & \\
\hline
\end{tabular}

Fuente: Elaborado por los autores.

Nota: * Valores calculados a partir de la $\mu$ del DC-Ame de cada medio analizado entre el número total de estos, por país analizado.

*** Valores calculados a partir de la $\mu$ del DC-Ame total de cada país entre el número de países analizados por región dentro del continente americano.

***⿰冫⿰亅⿱丿丶丶 Valores calculados a partir de la $\mu$ del DC-Ame total de cada región analizada entre el número de total de estas, dentro del continente americano.

$\wedge$ Nivel 1 = Bajo (DC-Ame $\leq 40$ ); Nivel $2=$ Medio (DC-Ame $>40 \leq 60$ ); Nivel $3=$ Alto (DC-Ame $>60$ ).

$\wedge \wedge$ DC-Ame $=$ Desarrollo de medios digitales en el continente americano 
posiciones adelantadas (nivel alto) dentro de este proceso; mientras que los países ubicados en Centroamérica y el Caribe (islas), en este orden, se ubican por detrás de este escenario digital.

Al momento de preguntarnos si existe una diferencia estadísticamente significativa entre el desarrollo de los medios digitales de los países de América, debemos indicar que para analizar ésta se aplicó una ANOVA de una vía, luego de comprobarse los supuestos de normalidad mediante las pruebas de KolmogorovSmirnov y Lilliefors (P-Valor 0,975) y la homocedasticidad mediante la prueba de Levene's (P-valor 0,752773).

La tabla IV, nos permite ver cómo la Razón-F, el cociente entre el estimado entre-grupos y el estimado dentro-de-grupos, de la ANOVA aplicada para ello, es igual a 1,89637. Puesto que el valor-P de la prueba-F es menor que 0,05, podemos decir que existe una diferencia estadística significativa entre la media del DC-Ame entre países, con un nivel del 95,0\% de confianza o, en otras palabras, se rechazaría la hipótesis de que las medias existentes en torno al desarrollo de los medios digitales son iguales en los diferentes países.

\section{TABLA IV}

Anova para indice de desarrollo de cibermedios (DC-AME), por país

\begin{tabular}{c|c|c|c|c|c}
\hline \multicolumn{1}{c|}{ Fuente } & $\begin{array}{c}\text { Suma de } \\
\text { cuadrados }\end{array}$ & G1 & $\begin{array}{c}\text { Cuadrado } \\
\text { medio }\end{array}$ & Razón-F & Valor-P \\
\hline Entre grupos & $4.852,89$ & 26 & 186,65 & 1,90 & 0,0152 \\
\hline Intra grupos & $8.267,68$ & 84 & 98,4248 & & \\
\hline Total (Corr.) & $13.120,6$ & 110 & & & \\
\hline
\end{tabular}

Fuente: Elaborado por los autores, según SPSS 18.

Nota: Número de observaciones (medios digitales analizados): 111.

Número de niveles: 27.

A partir del análisis factorial de correspondencia simple aplicado para identificar las posibles relaciones existentes entre las características regionales y los niveles de DC-Ame, se observa sobre un mapa factorial (figura 1) cómo los puntos más cercanos reflejan asociaciones entre categorías que enmarcan el nivel de desarrollo de los medios digitales según regiones existentes en América, así: el Nivel 3 (alto) más cercano a los medios analizados provenientes de los países de Norte y Sur América, los cuales presentan menores distancias entre sí; el nivel 2 (medio), más cercano a los países de Centroamérica; y el Nivel 1 (Bajo), más próximo al grupo de medios correspondientes a las islas del Caribe.

La ubicación de los niveles más altos del DC-Ame en los países de Norte y Suramérica, nos lleva a preguntarnos ¿Qué características de estos países influyen sobre el DC-Ame? A partir de los indicadores existentes en el Banco Mundial 
FIGURA 1

Nivel de DC-Ame, según regiones en el continente americano

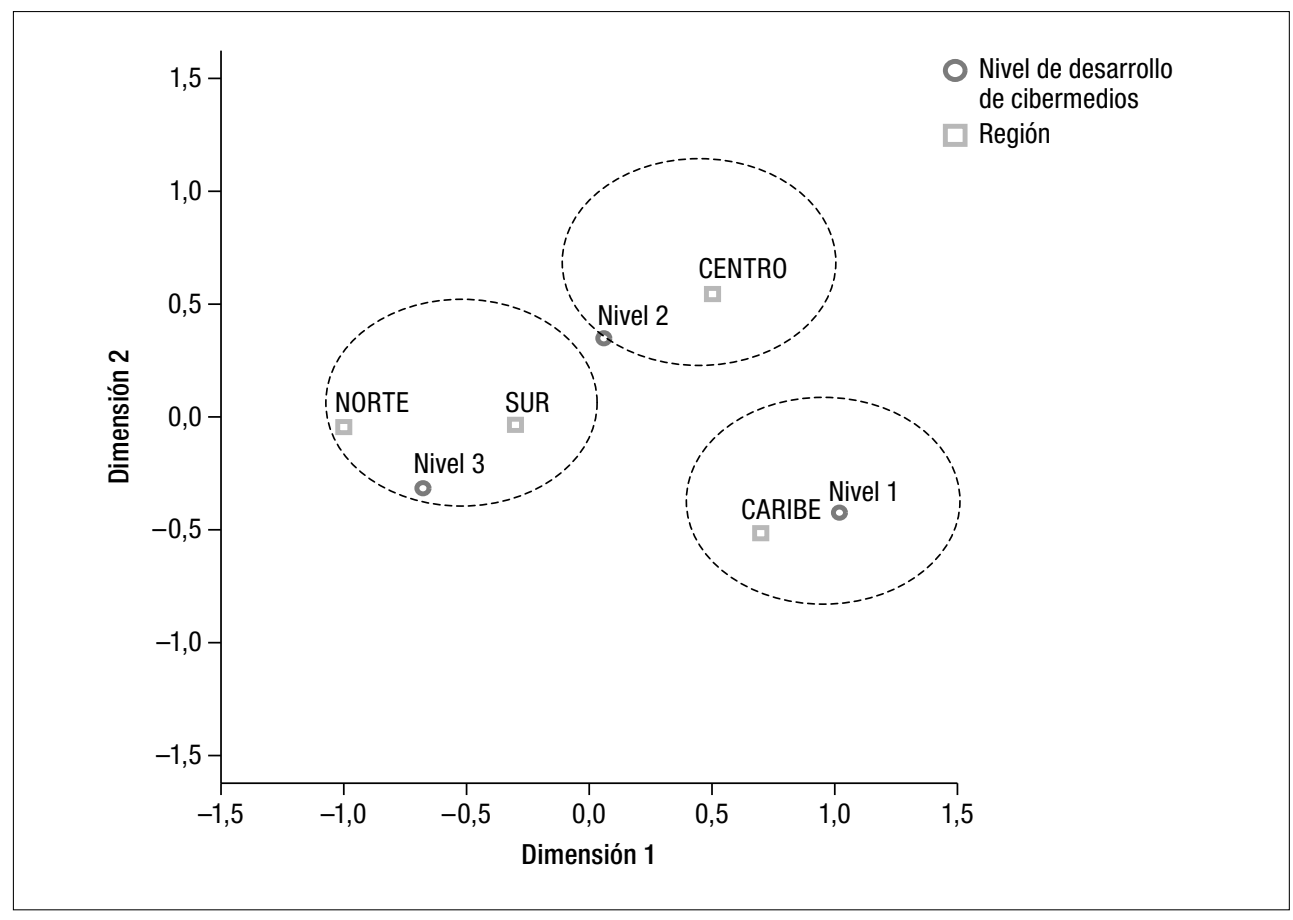

Fuente: Elaborado por los autores, a partir de análisis factorial de correspondencia simple entre DC-Ame e indicadores seleccionados del Banco Mundial (2008).

(http://datos.bancomundial.org/pais), vemos en la tabla V cómo los países asociados a menores niveles de desarrollo de DC-Ame son aquellos que presentan una mayor densidad de población (personas por kilómetro cuadrados), un mayor porcentaje de habitantes en zonas rurales y una mayor tasa de mortalidad; mientras que los países de las regiones con mayor nivel de desarrollo de medios digitales son aquellos que presentan mayores porcentajes de población urbana, un mayor ahorro interno bruto (como \% del PIB), un mayor número de abonados a Internet por banda ancha fija (por cada 100 personas), así como un mayor número de usuarios de Internet (por cada 100 personas).

Si bien la información destacada en la tabla $\mathrm{V}$ nos permite apreciar implicaciones de aspectos externos, propios de cada país donde se ubican los medios digitales analizados, en torno al tema tratado, ello no nos permite dilucidar las posibles relaciones directas ni de causalidad de los aspectos provenientes de dicho escenario en el desarrollo de los medios digitales en cada país y las diferentes regiones del continente americano observados hasta ahora. Con este fin, se evaluó la posible existencia de relaciones entre las condiciones existentes en cada 
TABLA V

Indicadores externos relacionados con los niveles del DC-Ame en el continente americano

\begin{tabular}{|c|c|c|c|c|}
\hline \multirow{2}{*}{ Indicador del Banco Mundial } & \multicolumn{4}{|c|}{ Región } \\
\hline & Caribe & Centro & Norte & Sur \\
\hline $\begin{array}{l}\text { Densidad de población (personas } \\
\text { por kilómetro cuadrado) }\end{array}$ & 169,36 & 111,68 & 31,28 & 25,44 \\
\hline $\begin{array}{l}\text { Tasa de fertilidad, total (nacimien- } \\
\text { tos por cada mujer) }\end{array}$ & 2,01 & 2,81 & 2,05 & 2,47 \\
\hline $\begin{array}{l}\text { Crecimiento de la población } \\
\text { (\% anual) }\end{array}$ & 0,73 & 1,57 & 1,07 & 1,21 \\
\hline $\begin{array}{l}\text { Población rural (\% de la pobla- } \\
\text { ción total) }\end{array}$ & 41,02 & 41,61 & 20,23 & 21,10 \\
\hline Población urbana (\% del total) & 58,98 & 58,39 & 79,77 & 78,90 \\
\hline $\begin{array}{l}\text { Crecimiento del PIB per cápita } \\
\text { (\% anual) }\end{array}$ & 1,73 & 2,57 & $(0,41)$ & 4,88 \\
\hline Ahorro interno bruto ( $\%$ del PIB) & 17,68 & 8,36 & 20,65 & 24,39 \\
\hline $\begin{array}{l}\text { Gasto de consumo final de los } \\
\text { hogares por crecimiento per cá- } \\
\text { pita ( } \% \text { anual) }\end{array}$ & 6,25 & 4,00 & 0,46 & 4,87 \\
\hline $\begin{array}{l}\text { Formación bruta de capital (\% del } \\
\text { crecimiento anual) }\end{array}$ & 9,01 & 4,85 & $(1,12)$ & 16,21 \\
\hline Crecimiento del PIB ( $\%$ anual) & 2,47 & 4,20 & 0,67 & 6,15 \\
\hline Población activa, total & $2.302 .701,38$ & $2.764 .411,50$ & $75.173 .770,00$ & $18.580 .052,40$ \\
\hline $\begin{array}{l}\text { Abonos a teléfonos celulares (por } \\
\text { cada } 100 \text { personas) }\end{array}$ & 84,38 & 86,57 & 74,42 & 88,17 \\
\hline $\begin{array}{l}\text { Abonados a Internet por banda } \\
\text { ancha fija (por cada } 100 \text { personas) }\end{array}$ & 4,61 & 1,89 & 20,56 & 4,30 \\
\hline $\begin{array}{l}\text { Usuarios de Internet (por cada } \\
100 \text { personas) }\end{array}$ & 31,56 & 16,27 & 57,37 & 26,24 \\
\hline $\begin{array}{l}\text { Tasa de mortalidad, menores de } \\
5 \text { años (por cada 1.000) }\end{array}$ & 20,84 & 23,42 & 10,77 & 22,76 \\
\hline $\begin{array}{l}\text { Riesgo de por vida de muerte ma- } \\
\text { terna }(\%)\end{array}$ & 0,16 & 0,30 & 0,09 & 0,24 \\
\hline $\begin{array}{l}\text { Tasa de mortalidad materna (es- } \\
\text { timado mediante modelo, por } \\
\text { cada } 100.000 \text { nacidos vivos) }\end{array}$ & 69,20 & 90,83 & 40,33 & 84,70 \\
\hline $\begin{array}{l}\text { Gasto en salud, sector público } \\
\text { (\% del PIB) }\end{array}$ & 4,34 & 4,33 & 5,62 & 3,46 \\
\hline
\end{tabular}

Fuente: Banco Mundial - Cálculos de los autores. 
país de América respecto al nivel de desarrollo de los medios digitales tomados como caso de estudio, a través de un modelo de regresión múltiple. Los datos observados en la tabla VI nos muestran cómo la única variable significativa dentro del modelo resultó ser el número de usuarios de Internet por cada 100 personas. En este sentido el modelo indica que el DC-Ame responde en forma positiva a los aumentos en el número de usuarios de Internet, en cuanto a la bondad de ajuste. El estadístico $\mathrm{R}^{2}$ indica que el modelo explica un 22,5\% de la variabilidad de los datos y el estadístico $\mathrm{R}^{2}$ corregido fue de $17,9 \%$, que es más conveniente para comparar modelos con diferentes números de variables independientes. Por otra parte, el p-valor obtenido del análisis de la tabla ANOVA del modelo es mucho menor que 0,05; por lo cual, podemos aceptar que la variable explicativa Usuarios de Internet (por cada 100 personas) tiene un efecto significativo sobre el DC-Ame al momento de intentar ayudar a responder al interrogante acerca de los posibles factores externos que pueden estar incidiendo en la evolución de los medios digitales en todo el continente americano.

TABLA VI

Modelo de regresión sobre incidencia de factores externos en el nivel de desarrollo de los medios digitales en América

\begin{tabular}{|c|c|c|c|c|c|c|}
\hline & \multirow[t]{2}{*}{ Modelo } & \multicolumn{2}{|c|}{$\begin{array}{c}\text { Coeficientes } \\
\text { no estandarizados }\end{array}$} & \multirow{2}{*}{$\begin{array}{c}\begin{array}{c}\text { Coeficientes } \\
\text { tipificados }\end{array} \\
\text { Beta } \\
\end{array}$} & \multirow[b]{2}{*}{$t$} & \multirow[b]{2}{*}{ Sig. } \\
\hline & & $\boldsymbol{B}$ & Error típ. & & & \\
\hline \multirow[t]{2}{*}{1} & (Constante) & 56,056 & 2,320 & & 24,164 & 0,000 \\
\hline & IT3 & 0,150 & 0,067 & 0,474 & 2,220 & 0,040 \\
\hline
\end{tabular}

Fuente: Elaborado por los autores.

Nota: a. Variable dependiente: DC-Ame.

IT3 = Usuarios de Internet (por cada 100 personas).

$R^{2}=0,225, R^{2}$ (Corregida) $=0,179 ;$ P-valor $($ Anova $)=0,040$.

\section{Conclusiones}

Los resultados expuestos nos llevan a plantear un estado alrededor de los medios digitales en el continente americano, durante 2011, en el que parecen existir diferencias marcadas entre los medios digitales que lideran este sector y que se ubican en las posiciones media o baja, los cuales representan el 81,98\% del total de los casos estudiados en este trabajo (91 de los 111 medios digitales). Por tanto, todo parece indicar que aun no están dadas las condiciones para trascender y hacer realidad el proceso de apropiación y transformación de las oportunidades que brinda Internet y las TIC, a pesar de las ventajas que pueden llevar consigo los escenarios virtuales que hoy están desarrollándose, como bien mencionan autores como Díaz-Noci (1997); Canga (2001); Cabrera (2001), Sala- 
verría y Sábada (2004); Bermejo (2005); Van Der Wurff y Lauf (2005); Salaverría (2005a, 2005b); Briggs (2007), Cardoso (2008); Gilin (2008), o Flores (2008, 2011), por citar solo algunos.

Si bien sabemos que varios de los medios más visitados en los países del continente americano están apostando por la calidad y la participación ciudadana, como es el caso de El Tiempo de Colombia (77/100 puntos), The NY Times de Estados Unidos (77/100 puntos), El Mercurio de Chile (75/100 puntos) y El País del Uruguay (75/100 puntos), la diferencia entre éstos y sus equivalentes con mayores retrasos en dichos temas, como el Diario Extra de Costa Rica (22/100 puntos), El Diario de Bolivia (26/100), Trinidad Newsday (27/100 puntos), las Últimas Noticias de Chile (32/100 puntos) y Fútbol Nica de Nicaragua (36/100 puntos), resulta clara. La puesta en evidencia de estas desigualdades puede contribuir a la formación de una hoja de ruta para los medios en el continente, especialmente si prestamos atención a los indicadores que tienen que ver con la participación activa de los usuarios y la profundización de la información en donde se observan las mayores carencias, en general.

Asimismo, podemos decir que existe en la actualidad un horizonte alrededor del desarrollo de los medios digitales en el continente americano; en donde, además de apreciarse la presencia de diferencias estadísticas significativas en torno a los niveles de desarrollo de estos medios entre países, podemos identificar de forma clara, con base a los medios digitales estudiados, que el desarrollo de este sector está siendo liderado, no sólo, por países como Estados Unidos, Canadá y México (países de Norteamérica), sino por países provenientes del resto de regiones que conforman dicho continente, como por ejemplo: Perú, Colombia, Brasil, Honduras, Uruguay, Paraguay o República Dominicana. Ello pese a las diferencias estadísticas observadas en la media de los niveles de desarrollo de los medios digitales analizados en todos los países estudiados a nivel continental. Por tanto, nos llevaría a señalar que al hablar de este proceso en América, la temática no sólo debería tomar en consideración los avances generados desde los principales medios de comunicación digitales provenientes de los países con mayores niveles de desarrollo económico y social en el continente, según los datos dispuestos por el Banco Mundial (http://datos.bancomundial. org/indicador) sino también de otros que, a pesar de las diferentes condiciones sociales y económicas que poseen, también lideran este fenómeno.

Los datos expuestos aquí también ayudan a dar fortaleza a lo expuesto por Said y Arcila (2011), por ejemplo, en torno a los puntos fuertes y debilidades que presentan los medios digitales de América Latina. Pese a que nuestro estudio amplía el rango de análisis de dichos autores, se logra reafirmar lo observados por ellos en lo que se refiere a la fortaleza que presentan los medios digitales y en lo concerniente al uso de las Web 2.0 de forma generalizada. Ello, en medio de un contexto caracterizado también por un conjunto de debilidades como el que se refiere a la accesibilidad de los portales, las posibilidades de profundización de la información difundida en estos medios y el empleo de herramientas de interacción dispuestos por los medios a sus usuarios, en general. 
Si bien el análisis de los datos tomados en consideración para el desarrollo de este artículo nos marca unos rasgos característicos entre países adelantados (alto nivel de DC-Ame) o no (bajo nivel de DC-Ame), el único aspectos que incide estadísticamente fuera del sector donde se lleva a cabo este proceso estudiado (medios de comunicación) termina siendo la densidad de usuarios conectados a Internet en los países del continente analizados en este artículo. Por tanto, podemos decir que, al menos uno de los factores que hemos logrado determinar que inciden en el rumbo que tomen los medios digitales a nivel continental, resulta ser la capacidad que tenga cada uno de ellos por lograr solventar las brechas tecnológicas que puedan estar presentando, al momento de permitir a sus ciudadanos acceder de forma frecuente o no a Internet.

Pese a sólo lograr identificar un único factor externo en el marco de este trabajo, la densidad de usuarios conectados a Internet, que incide de forma estadísticamente significativa en el desarrollo de los medios digitales, logran explicar casi un $18 \%$ la variabilidad de los datos obtenidos en el índice de DC-Ame de los países analizados. Ello nos lleva a reafirmar la necesidad de seguir avanzando en procesos de investigación que permitan visualizar mejor otros factores y que ayuden a dar fortalezas a las diferentes estrategias y planes relacionados con el desarrollo de los medios digitales y contenidos interactivos a ser expuestos desde estos escenarios.

Los datos mostrados, además, nos permiten tipificar, según el nivel de desarrollo de los medios digitales, al continente americano por región. Siendo las regiones de Norte y Suramérica las que poseen un nivel de desarrollo alto de cibermedios, de acuerdo con el índice construido para su medición ; mientras que Centroamérica y las islas del Caribe se encuentran en niveles de avance medio y bajo respectivamente en dicha materia. Por ello, estimamos pertinente hacer uso de estos datos para, no sólo, adquirir una visión general de las fortalezas y debilidades presentes en torno al tema propuesto, sino también ayudar a establecer algunas recomendaciones que pudiesen ayudar a reducir las carencias observadas, como por ejemplo: 1) que la mayoría de los medios digitales ajusten los diseños de sus portales con el fin de garantizar la accesibilidad a una mayor cantidad de usuarios, siguiendo las normas W3C (http://www.w3c.es/ traducciones/es/wai/intro/accessibility) , por ejemplo; 2) que mejoren los procedimientos de indexación de los contenidos publicados en buscadores, ayudando así a un aumento de su visibilidad y acceso de estos desde Internet para un mayor número de usuarios; 3) capacitar a los profesionales que ejercen la función periodística en los medios, para que les permita hacer un uso más intensivo de los hipervínculos y de las oportunidades que brinda Internet al momento de desarrollar contenidos que fortalezcan un historial alrededor de una noticia desde un escenario multiformato; 4) revisar los procedimientos internos de admisión de herramientas de interacción entre el medio-usuario-periodista, que faciliten una comunicación más directa entre dichos actores y el fortalecimiento del rol "prosumidor" que tienen los usuarios en la actualidad (Toffler, 1980), es decir, usuarios con una creciente capacidad no sólo de consumir con- 
tenido sino de producirlos también, y 5) a nivel de todo el continente, favorecer mecanismos que ayuden a la universalización del servicio de Internet en la mayoría de los países analizados, favoreciendo con ello el fortalecimiento de un contexto externo que ayude al desarrollo de los medios digitales en el continente americano.

\section{Bibliografía}

Bermejo, L. (2005): El Informe sobre la Situación Laboral y Profesional del Periodista Digital en Cataluña. Mediatika, vol. 11, 171-183.

Briggs, M. (2007): Periodismo 2.0. Una guía de alfabetización digital. (1. ${ }^{a}$ ed.) Estados Unidos: Knight Foundation, http://knightcenter.utexas.edu/Periodismo_20.pdf [5 de febrero de 2011].

Boyer, D. (2010): Digital Expertise in Online Journalism (And Anthropology). Anthropological Quarterly, vol. 83 (1), 73-95.

Cabrera, M. A. (2001): Convivencia de la prensa escrita y la prensa "on line» en su transición hacia el modelo de comunicación multimedia». Estudios sobre el mensaje periodístico, vol. 7. 71-78.

Canga, J. (2001): Periodismo e Internet: nuevo medio, vieja profesión. Estudios sobre el mensaje periodístico, vol. 7, 33-48.

Cardoso, G. (2008): Los medios de comunicación en la Sociedad en Red. Barcelona: Editorial UOC, p. 576.

Castells, M. (2000). Internet y la Sociedad en Red. "Lliçó inaugural del programa de doctorat sobre la societat de la informació i el coneixement». Barcelona: Universidad Oberta de Catalunya, http://www.uoc.edu/web/cat/articles/castells/castellsmain1.html [21 de abril de 2006].

Constanza-Chock, S. (2008): The inmigrant Right Movement on the Net: Between "Web2.0" and Comunicación Popular. American Quaterly, vol. 60 (3), 851-864.

Díaz-Noci, J. (1997): Tendencias del periodismo electrónico. Una aproximación a la investigación sobre medios de comunicación en Internet. Revista Zer, vol. 2, www.ehu.es/ zer/zer2/6artdiaz.html [5 de diciembre de 2010].

Fidler, R. (1998): Mediamorfosis. Madrid: Granica, p. 439.

Flores, J. (2008): El «Blog Band» de la información. En: Jesús F.; Cebrián, M., y Estévez, F. (ed.) Blogalaxia y periodismo en la Red. Estudios, análisis y reflexiones. Fragua, Madrid, España.

Flores, J. (2011): Reinventar el Periodismo y los Medios. Madrid: Fragua, p. 254.

García de Torres, E.; Lyudmyla, Y.; Rost, A.; Calderín, M.; Rojano, M.; Concha, E.; Said, E.; Jerónimo, P.; Arcila, C.; Serrano, A.; Badillo, J., y Corredoira, L. (2011): See you on Facebook or Twitter? The use of social media by 27 news outlets from 9 regions in Argentina, Colombia, Mexico, Peru, Portugal, Spain and Venezuela. 12 international Symposium on Online Journalism. Texas: Estados Unidos: Universidad de Texas.

Gilin, P. (2008): New Media, New Influencers and Implications for Public Relations. Journal of New Communications Research and Institute for PR, vol. II (2), 1-10. 
Hart, K. (2009): An Anthropologist in the World Revolution. Anthropology Today, vol. 25 (6), 24-25.

Lewis, C., y Fabos, B. (2005): Instant Messaging Literacies, and Social Identities. Reading Research Quaterly, vol. 20 (4), 470-501.

McCombs, M.; Einsiedel, E., y Hugh, D. (1991): Contemporary public opinion. Estados Unidos: Routledge, p. 114.

Palacios, M., y Díaz-Noci, J. (2009): On-line Journalism: Research Methods. A Multidisciplinary Approach in Comparative Perspective. Bilbao: Servicio Editorial de la Universidad del País Vasco, p. 180.

Palacios, M. (coord.) (2011): Ferramentas para Análise de qualidade no ciberjornalismo. Vol. 1, Modelos. Portugal: LabCom Books, p. 292.

Piscitelli, A. (2002): Cibercultura 2.0. En la Era de las Máquinas Inteligentes. Buenos Aires: Paidós, p. 288.

Resina, J. (2010): Ciberpolítica, redes sociales y nuevas movilizaciones en España: el impacto digital en los procesos de deliberación y participación ciudadana. Mediaciones Sociales, vol. 11, 143-164.

Rodríguez-Martínez, R.; Codina, Ll., y Pedraza-Jiménez, R. (2010): Medios digitales y web 2.0: modelo de análisis y resultados de aplicación. El Profesional de la Información, vol. 19 (1), 35-44.

Said-Hung, E., y Arcila-Calderón, C. (2011): Los cibermedios en América Latina y las web 2.0. Comunicar, vol. XIX(37), 125-131.

Said-Hung, E.; Arcila-Calderón, C., y Mendez-Barraza, J. (2011): Desarrollo de los Cibermedios en Colombia. El Profesional de la Información, vol. 20(1), 47-53.

Salaverría, R. (2005a): Redacción periodística en Internet. Pamplona: EUNSA, p. 184.

Salaverría, R. (coord.) (2005b): Cibermedios. El impacto de Internet en los medios de comunicación en España. Sevilla: Comunicación Social Ediciones y Publicaciones, p. 339.

Salaverría, R., y Sádaba, Ch. (coord.) (2004): Towards new media paradigms: content, producers, organizations and audiences. II International Conference of COST A2O. Pamplona, España. Junio 27-28, en Pamplona: España: Universidad de Navarra.

Toffler, A. (1980): La tercera Ola. Bogotá: Plaza \& Janés, p. 339.

Turner, F. (2005): Where the Counterculture Met the New Economy: The WELL and the Origins of Virtual Community. Technology and Culture, vol. 46 (3), 485-512.

Van Der Wurff, R., y Lauf, E. (2005): Print and online newspapers in Europe. A comparative analysis in 16 countries. Amsterdam: Het Spinhuis Publishers, p. 326.

Zamith, F. (2008): A Methodological Proposal to Analyze the News Websites use of the Potentialities of the Internet. 9th International Symposium on Online Journalism. Texas: Estados Unidos: Universidad de Texas. 